\title{
Mesenchymal stem cells show little tropism for the resting and differentiated cancer stem cell-like glioma cells
}

\author{
ZHENLIN LIU ${ }^{1}$, ZHONGMIN JIANG $^{2}$, JIANYONG HUANG $^{4}$, SHUQIANG HUANG $^{4}$, \\ YANXIA LI ${ }^{3}$, FENG SHENG ${ }^{3}$, SIMIAO YU ${ }^{1}$, SHIZHU YU ${ }^{5-7}$ and XIAOZHI LIU ${ }^{1,3}$ \\ Departments of ${ }^{1}$ Neurosurgery, ${ }^{2}$ Pathology, ${ }^{3}$ The Central Laboratory, The Fifth Central Hospital of Tianjin, Tianjin, \\ P.R. China; ${ }^{4}$ Department of Biomedical Engineering, Duke University, Durham, NC, USA; ${ }^{5}$ Tianjin Neurological Institute, \\ Tianjin Medical University General Hospital, ${ }^{6}$ Key Laboratory of Post-trauma Neuro-repair and \\ Regeneration in Central Nervous System, Ministry of Education; ${ }^{7}$ Tianjin Key Laboratory of Injuries, \\ Variations and Regeneration of Nervous System, Tianjin, P.R. China
}

Received November 27, 2013; Accepted January 11, 2014

DOI: 10.3892/ijo.2014.2284

\begin{abstract}
Intrinsic resistance of glioma cells to radiation and chemotherapy is currently hypothesized to be partially attributed to the existence of cancer stem cells. Emerging studies suggest that mesenchymal stem cells may serve as a potential carrier for delivery of therapeutic genes to disseminated glioma cells. However, the tropism character of mesenchymal stem cells for cancer stem cell-like glioma cells has rarely been described. In this study, we obtained homologous bone marrow-derived (BM-) and adipose tissue-derived (AT-) mesenchymal stem cells (MSCs), fibroblast, and cancer stem cell-like glioma cells (CSGCs) from tumor-bearing mice, and compared the tropism character of BM- and AT-MSCs for CSGCs with various form of existence. To characterize the cell proliferation and differentiation, the spheroids of CSGCs were cultured on the surface of the substrate with different stiffness, combined with or withdrew basic fibroblast growth factor (bFGF) and epidermal growth factor (EGF) in medium. Our results showed that the CSGCs during the process of cell proliferation, but not in resting and differentiated status, display strong tropism characteristics on both BM- and AT-MSCs, as well as the expression of their cell chemokine factors which mediate cell migration. If the conclusion is further confirmed, it may expose a fatal flaw of MSCs as tumor-targeted delivery of therapeutic agents in the treatment of the CSGCs, even other cancer stem cells, because there always exist a part of cancer stem cells that are in resting status. Overall, our findings provide novel insight into the
\end{abstract}

Correspondence to: Professor Xiaozhi Liu, Department of Neurosurgery, The Fifth Central Hospital of Tianjin, 41 Zhejiang Road, Tanggu District, Tianjin 300450, P.R. China

E-mail: 1xz7997@126.com

Key words: cancer stem cell, glioma, mesenchymal stem cells, stem cells complex issue of the MSCs as drug delivery in the treatment of brain tumors, especially in tumor stem cells.

\section{Introduction}

Glioblastoma multiforme (GBM) is one of the most frequent and devastating primary brain tumors in adults with a high rate of death and disability $(1,2)$. The WHO grade III anaplastic astrocytomas and grade IV GBM make up approximately three-quarters of all gliomas. GBMs make up 17\% of all primary brain tumors in the United States. Despite both the knowledge of brain tumor biology and current resources for GBM treatment have greatly developed over the past decade, a large fraction of brain cancer patients suffer tumor recurrence shortly after GBM tumor resection. The current 5- and 10-year survival rates for GBM patients are 4.5 and $2.7 \%$, respectively (3). Therefore, new and more effective therapies are necessary to improve the prognosis of this disease.

Brain tumor recurrence due to the resistance to chemo- and radiotherapy of the cancer cells. Cells that escape chemotherapy- and radiotherapy-induced cell death eventually re-enter the cell cycle and contribute to local tumor recurrence $(4,5)$. The identification and significance of cancer stem cell-like glioma cells (CSGCs) reveal the mysterious mask of drug resistance and tumor recurrence. Most of the CSGCs are in the resting state, namely the G0 phase of the cell cycle, and express ATP-binding cassette transporters that detoxify cells by eliminating exogenous compounds (6-10). Therefore, understanding the mechanisms associated with 'escape' from clinical therapy of tumor stem cells, in conjunction with the use of new treatments based on the clearance and intervention of tumor stem cells, are required to advance the treatment of glioma.

The use of mesenchymal stem cells (MSCs) as therapeutic vehicles for brain tumors has garnered much attention over the past decade. This is attributable to the fundamental ability of MSCs to migrate, or home, to brain tumors irrespective of the blood-brain barrier and to be manipulated into expressing various therapeutic molecules, such as interleukins, inter- 
ferons, prodrugs, oncolytic viruses, antiangiogenic agents, pro-apoptotic proteins and growth factor antagonists (11-14). A number of studies have shown that MSCs express both chemokine receptors and adhesion molecules enabling their homing function to target sites in vivo in response to specific chemokine gradients. Many cytokine/receptor pairs, such as SDF-1/CXCR4, SCF/c-Kit, HGF/c-Met, VEGF/VEGFR, PDGF/PDGFr, MCP-1/CCR2, and HMGB1/RAGE (15-18), play roles in inducing stem cells recruitment to tumors.

Although emerging studies suggest that MSCs may serve as therapeutic vehicles for the treatment of various tumors, the tropism character of mesenchymal stem cells for cancer stem cells has rarely been described. In this study, we tested the tropism of bone marrow-derived (BM-) and adipose tissue-derived (AT-) mesenchymal stem cells (MSCs) for cancer stem cell-like glioma cells (CSGCs). In order to obtain optimal results, we obtained BM- and AT-MSCs, fibroblast cells, and CSGCs from homologous tumor-bearing mice. To our surprise, there were almost no migration of MSCs, including BM- and AT-MSCs, towards CSGCs that were in the resting state, compared with fibroblast cells. However, the number of migrated MSCs was noticeably increased with the proliferation of CSGCs from the resting state into the cell cycle. In order to further explore the potential of MSCs migration for CSGCs, we also induced CSGCs differentiation into the terminal cells. The results displayed that the MSC migration was decreased significantly when the CSGCs were induced into terminal differentiation cells. The results were confirmed by further experiments from analysis of three important cytokine/receptor pairs, SCF/c-Kit, SDF-1/CXCR4 and VEGF/VEGFR, which play key roles in mediating the recruitment from MSCs to tumors. Unexpectedly, fibroblast, as a negative control, also showed a slight curve change, with the proliferation of CSGCs. This finding seems to be contradictory with many previous reports, which claimed fibroblasts have hardly any tropism for tumor cells (19). We speculate that fibroblasts may be involved in the stem cell migration as a helper cell component.

In the present study, we report for the first time that BM- and AT-MSCs show little migration towards CSGCs that were in the resting and differentiated status. Only when induced into proliferation process, CSGCs secrete chemokines and induce migration of MSCs. Our findings expose a fatal defect of MSCs-based tumor therapy, because there always exist a part of cancer stem cells that are in resting status, and bring new challenge for MSCs as vehicles of therapeutic agents in the treatment of the CSGCs, and even other tumor stem cells.

\section{Materials and methods}

Animals and the brain tumor model. All animal studies were done in accordance with institutional guidelines under the approved protocols. For the intracranial xenografts of glioma, male Sprague-Dawley rats (2-week old; Animal Center of the Chinese Military Medical Sciences) were anesthetized with ketamine/xylazine i.p. and stereotactically inoculated with $4 \times 10^{5}$ C6 cells (Tianjin Institute of Neurology) suspension (in $5 \mu \mathrm{l}$ ) into the right frontal lobe $(1.5 \mathrm{~mm}$ lateral and $1 \mathrm{~mm}$ anterior to bregma, at $2.5 \mathrm{~mm}$ depth from the skull base) using a stereotactic apparatus with a microinfusion pump. The needle was maintained in place for $\geq 3$ min following injection and the incision was then sutured. MRI was conducted at the 7th day after cell transplantation at MRI Testing Center of The Fifth Central Hospital of Tianjin.

\section{Homologous cell culture}

Cancer stem cell-like glioma cells. Glioblastoma tissue was obtained from the confirmed tumor-bearing rats. Glioblastoma blocks were rinsed twice with D-Hanks solution, and then sheared into paste after vessels and necrotic tissue were eliminated. After it was digested with $0.25 \%$ trypsin (Invitrogen, Carlsbad, CA, USA), and cells were pipetted into a single cell suspension. Digestion was terminated using $10 \%$ fetal bovine serum (Hangzhou, Sijiqing, China). Tissues were filtered through a $30-\mu \mathrm{m}$ mesh and centrifuged at $1,000 \mathrm{rpm}$ for $5 \mathrm{~min}$. After removal of the supernatant, cells were collected and counted. Cells $\left(1 \times 10^{6}\right)$ were suspended with PBE incubation solution (including $0.5 \%$ bovine serum albumin, $0.08 \%$ EDTA in PBS, $\mathrm{pH} 7.2$ ) to a final concentration of $1 \times 10^{8}$ cells in $0.5 \mathrm{ml}$, then incubated with anti-CD133 antibody (antibody final concentration $20 \mu \mathrm{g} / \mathrm{ml}$ ) at $4^{\circ} \mathrm{C}$ for $30 \mathrm{~min}$ and then with antibody-coated superfine magnetic beads (Miltenyi Biotec) at $10^{\circ} \mathrm{C}$ for $15 \mathrm{~min}$ and suspended with 20 times volume of PBE solution. The separation column was installed into a magnetic field and pretreated with $0.5 \mathrm{ml} \mathrm{PBE}$, which was naturally eluted due to gravity. The incubated cell suspension was added to the separation column and naturally eluted, then $0.5 \mathrm{ml}$ PBE was added to the separation column and naturally eluted, the column was rinsed twice and separated from the magnetic field. The column was then inserted into a new tube, and 1-2 ml PBE was administered along the needle core, to remove the CD133 positive cells. The CSGCs spheres were cultured in Neurobasal medium (Invitrogen) containing 1xB27 (Invitrogen), $2 \mathrm{mM}$ L-glutamine, $30 \mathrm{U} / \mathrm{ml}$ penicillinstreptomycin, $1 \mathrm{U} / \mathrm{ml}$ heparin (Sigma), $20 \mathrm{ng} / \mathrm{ml}$ basic fibroblast growth factor (bFGF, Miltenyi Biotec) and $20 \mathrm{ng} / \mathrm{ml}$ epidermal growth factor (EGF, Provitro).

BM-MSCs. BM-MSCs were derived from long bone of hind legs in the same tumor-bearing rats as described previously (20). Briefly, the long bone of hind legs were dissected and bone marrow plugs were extracted from the bones by flushing the bone marrow cavity with complete culture medium. The cells were centrifuged $(1,400 \mathrm{rpm}, 8 \mathrm{~min})$, resuspended in complete culture medium, plated $\left(50 \times 10^{6}\right.$ cells per $75-\mathrm{cm}^{2}$ culture flask), and incubated at $37^{\circ} \mathrm{C}$ humidified atmosphere with $5 \% \mathrm{CO}_{2}$. Non-adherent cells were removed after $24 \mathrm{~h}$ and the culture medium was replaced every 3 days. Adherent cells reached confluence of 90-95\% within 10-15 days and were passaged with $0.25 \%$ trypsin (Invitrogen) in the ratio 1:3. Cells at passages 3 were identified by flow cytometric analysis to detect the expression of surface antigen CD29, CD31, CD34, CD45, CD71 and CD105 (Chemicon).

AT-MSCs. AT-MSC were isolated from subcutaneous fat tissue in the same tumor-bearing mice as described previously (21). Adipose tissue was cut into fine pieces, and digested with $1 \mathrm{mg} / \mathrm{ml}$ collagenase IA (Sigma) for $1 \mathrm{~h}$ at $37^{\circ} \mathrm{C}$ with shaking at a speed of $200 \mathrm{rpm}$. The released cells were centrifuged 
for $15 \mathrm{~min}$ at $400 \mathrm{x} \mathrm{g}$ to removed them from the tissues. The cell pellet suspended with PBS was filtered through a $70-\mu \mathrm{m}$ mesh to remove debris and centrifuged for $5 \mathrm{~min}$ at $1,500 \mathrm{rpm}$ each time to wash the cells. The residual cells were cultured and identified similarly to the BM-MSCs. The CD29, CD34, CD45, CD71, CD90 and CD105 (Chemicon) were selected for the flow cytometric analysis.

Fibroblast cells. The tails $(4-5 \mathrm{~cm})$ were harvested from the same tumor-bearing rats and rinsed with PBS three times. The tails were cut into $1 \mathrm{~mm}$ pieces, then were rinsed and cultured in DMEM supplemented with L-glutamine, 4,500 mg/l D-glucose, $10 \% \mathrm{FBS}$, and $1 \%$ antibiotic-antimycotic solution. All cells were incubated at $37^{\circ} \mathrm{C}$ in an incubator in a $5 \% \mathrm{CO}_{2} / 95 \%$ air atmosphere. After 5 days, tail pieces were discarded and the fibroblasts were continued in culture until they reached $90 \%$ confluency. Then $8 \times 10^{5}$ fibroblasts were taken for retroviral infection.

Preparation and characterization of the substrate. In order to finite the cells in the resting, proliferation, and differentiated status, the spheroids of CSGCs were cultured on the surface of the substrate with different stiffness, combined with or withdrew bFGF and EGF in medium. The sheets of polyacrylamide gel were prepared as described by Dembo and Wang (22). With varied stiffness, the gel here contained a mixture of acrylamide (10\%, Sigma) and different percents of bis-acrylamide $(0.03 \%$ for soft and $0.26 \%$ for stiff, respectively). The thickness of the polyacrylamide thin film was $60 \mu \mathrm{m}$, and the stiffness was tested as described by Qin et al (23) and Huang et al (24). For cell culture, the gel was incubated with type I collagen solution (0.2 mg/ml, c8919, Sigma-Aldrich) at $4^{\circ} \mathrm{C}$ overnight sterilized with UV irradiation.

CSGC proliferation and differentiation. The CSGC spheres with 100-200 cells each sphere were divided into three groups. The first part of the CSGC spheres were cultured on surface of the soft gel culture dishes with the Neurobasal medium containing bFGF and EGF. To limit the CSGCs in the resting status, the second part of the CSGC spheres were seeded on surface of the soft gel culture dishes with the same Neurobasal medium but lacking bFGF and EGF. To induce CSGC differentiation, the third part of the CSGC spheres were seeded on surface of the stiff gel culture dishes with the same Neurobasal medium but lacking bFGF and EGF. The size and morphology of CSGC spheres were observed. Twenty-four hours after culture, the resulting conditioned media of the three parts were collected, respectively, as chemoattractants for the testing of enzyme linked immunosorbent assay (ELISA) and transwell migration assay. The proliferation activity of cells was detected by immunocytochemistry for Ki-67, and the cell differentiation was detected by telomeric repeat amplification protocol assay and immunocytochemistry for the expression of CD133, nestin, GFAP, $\beta$-tubulin III, and myelin.

Telomeric repeat amplification protocol assay. Telomeric repeat amplification protocol assay was carried out using Telomerase PCR kit (Roche) following the manufacturer's recommendations and previously described (25).
Immunocytochemistry. The proliferation activity of cells in each group was detected by binding the monoclonal antibody $\mathrm{Ki}-67$ to a nuclear antigen present in all cells that are in the G1, S, G2, and M phase of the cell cycle, but not in the G0 phase. The cell differentiation was detected by immunocytochemistry for markers of undifferentiated CSGCs (CD133 and nestin), and differentiated CSGCs (GFAP for astrocyte, $\beta$-tubulin III for neuron, and myelin for oligodendrocyte). Immunocytochemistry was performed as described previously (26). 96-wells were fixed with $2 \%$ paraformaldehyde for $15 \mathrm{~min}$ at room temperature, treated with $5 \%$ normal goat serum (Vector Laboratories), and then stained with the following antibodies: anti-Ki-67 (mouse monoclonal IgG1; 1:2,000; Santa Cruz), anti-CD133 (mouse monoclonal IgG1; 1:1,000; Miltenyi Biotec), anti-nestin (mouse monoclonal IgG1; 1:2,000; Chemicon), anti- $\beta$-tubulin III (mouse monoclonal IgG1; 1:2,000; Chemicon), anti-GFAP (rabbit polyclonal; 1:1,000; Chemicon), and anti-myelin (mouse monoclonal; 1:1,000; Chemicon). The primary antibodies were detected with Tex-Red or FITC-conjugated donkey anti-mouse $\mathrm{IgG}$ and FITC-conjugated donkey anti-rabbit IgG (1:1,000; Jackson ImmunoResearch). Cells were counterstained with DAPI (Vector Laboratories). Images from 10 random fields per well were captured. The percentage was calculated based on the total number of nuclei counted.

ELISA for secreted cytokines by CSGCs. SCF, SDF-1 and VEGF proteins secreted into the culture supernatants were analyzed by ELISA accordance with the instructions. Briefly, $100 \mu 1$ of each standard and sample was added into appropriate wells and incubate overnight at $4^{\circ} \mathrm{C}$ with gentle shaking. After washing, $100 \mu \mathrm{l}$ of biotinylated antibody was added to each well for $1 \mathrm{~h}$ at room temperature. Then $100 \mu \mathrm{l}$ of prepared streptavidin solution was added to each well, and incubated for $45 \mathrm{~min}$ at room temperature. At last, $100 \mu \mathrm{l}$ of TMB One-Step Substrate Reagent (Item H) was added to each well for $30 \mathrm{~min}$ at room temperature in the dark with gentle shaking. After stoping the reaction, the result was read at $450 \mathrm{~nm}$ immediately.

Western blotting for expressed receptors by BM- and $A T$-MSCs. BM-MSCs or AT-MSCs or fibroblast cells were cultured, respectively, in the conditioned media of the three groups containing chemoattractants for $24 \mathrm{~h}$. The expression level of receptor, c-Kit, CXCR4 and VEGFR, was detected by western blotting as previously described (27). Proteins were incubated with primary antibodies against c-Kit $(1: 2,000$, Santa Cruz), CXCR4 (1:1,000, Santa Cruz), and VEGFR (1:1,000, Zhongshan Bio Corp.) followed by incubation with an HRP-conjugated secondary antibody (Zymed). The specific protein was detected using a SuperSignal protein detection kit (Pierce). The membrane was stripped and re-probed with a primary antibody against $\beta$-actin $(1: 1,000$, Santa Cruz).

In vitro migration studies. The migratory ability of BM- and AT-MSCs was determined using Transwell plates (Corning Costar) that were $6.5 \mathrm{~mm}$ in diameter with $8 \mu \mathrm{m}$ pore filters. BM- or AT-MSCs $\left(1 \times 10^{4}\right)$ were suspended in serum-free medium containing $0.1 \%$ bovine serum albumin (Sigma) and 

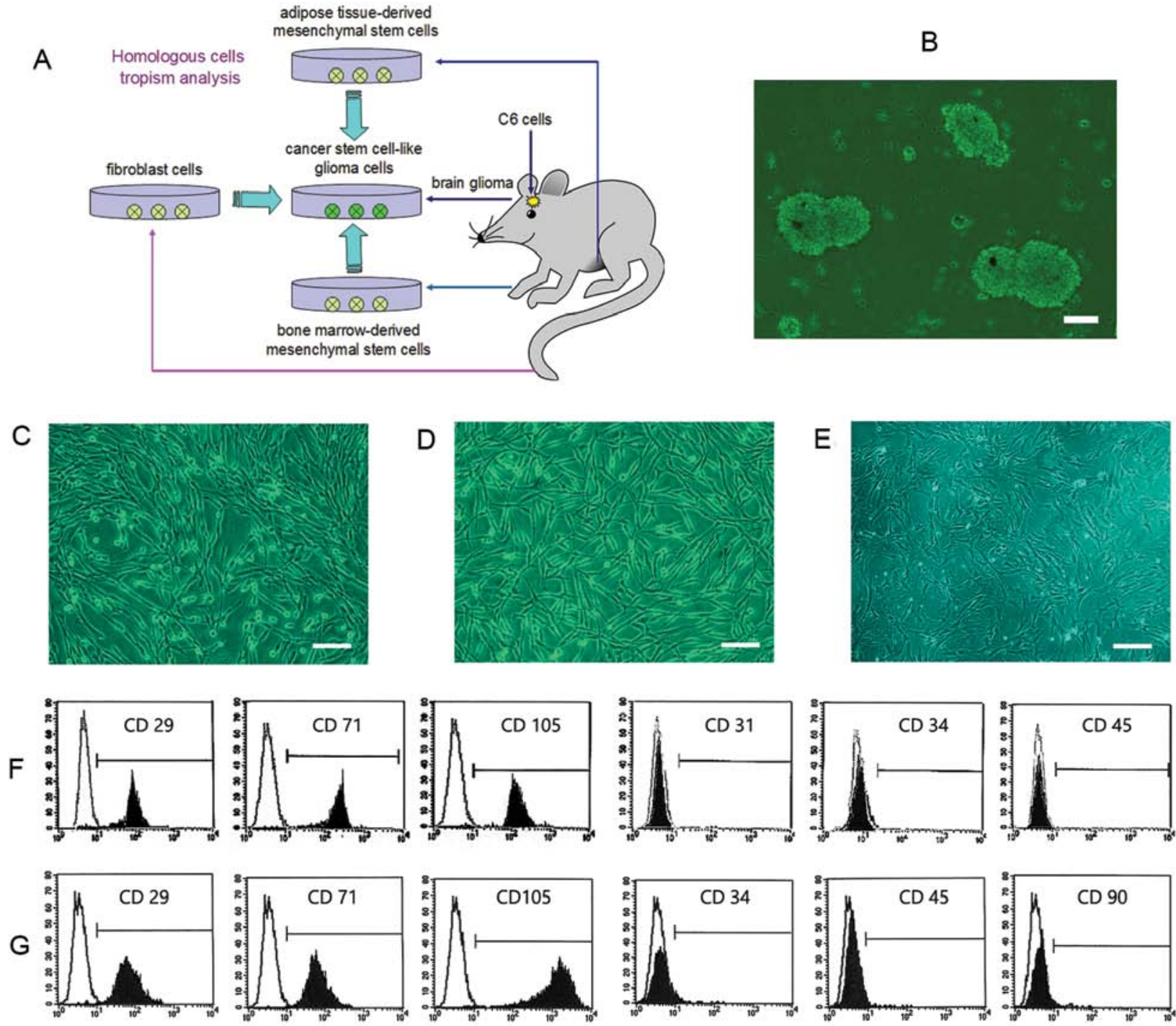

Figure 1. Characterization of homologous cell lines from the same rat. (A) A schematic drawing shows that all four cell lines, the glioma cells, BM-MSCs, AT-MSCs, and fibroblasts, were obtained from different regions of the same rat. (B) CSGC spheres were obtained from a rat confirmed to bear a tumor. (C) BM-MSCs proliferated rapidly to form a homogeneous population of spindle-shaped cells. (D) The growth characteristics of AT-MSCs were similar to those of BM-MSCs. (E) Compared to BM- and AT-MSCs, fibroblasts exhibited a relatively slow growth rate and flat cell shape. (F) Flow cytometric analysis revealed that BM-MSCs were positive for the typical mesenchymal markers CD29, CD71, and CD105 but negative for CD31, CD34 and CD45. (G) AT-MSCs were positive for the markers CD29, CD71, and CD105, but negative for CD34, CD45, and CD90 (bars, $15 \mu \mathrm{m})$.

seeded into the upper well, the fibroblast cells as negative control; $600 \mu 1$ of conditioned medium from the above was placed in the lower well of the Transwell plate, the Neurobasal medium containing or lacking bFGF and EGF as control. Following incubation for $24 \mathrm{~h}$ at $37^{\circ} \mathrm{C}$, cells that had not migrated from the upper side of the filters were removed with a cotton swab, and filters were stained with the Three-Step Stain Set (Diff-Quik; Sysmex). The number of cells that had migrated to the lower side of the filter was counted under a light microscope with five high-power fields (x400). Experiments were done in triplicate.

Statistical analysis. All data are expressed as mean $\pm \mathrm{SE}$. Statistical differences between different test conditions were determined by Student's t-test. $\mathrm{P}<0.05$ was considered statistically significant.

\section{Results}

Brain tumor model and MRI. The tested rats were listless and had reduced activity at 7 days following cell transplantation. MRI images showed intracranial space-occupying lesions (data not shown). The results from HE staining showed obvious atypical cells in the brain tissue (data not shown).

Characterization and identification of homologous cell lines. Following seeding in mesenchymal culture medium, typical fibroblastoid colonies could be detected after a few days rapidly forming a monolayer of adherent cells from both BM- and AT-MSCs. MSCs cultures contained a homogeneous population of spindle-shaped cells (Fig. 1C and D). Flow cytometric analysis revealed that BM-MSCs were positive for the typical mesenchymal markers CD29, CD71 and CD105 
A

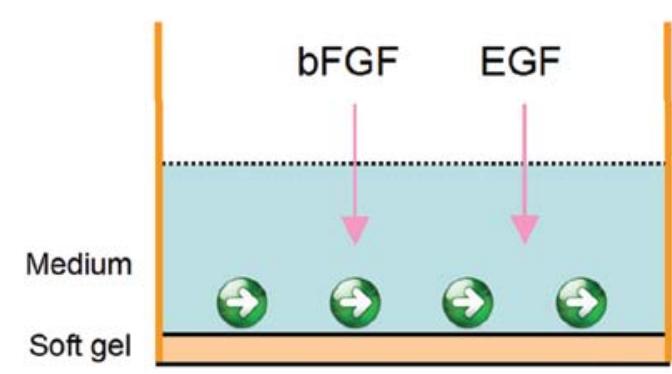

B

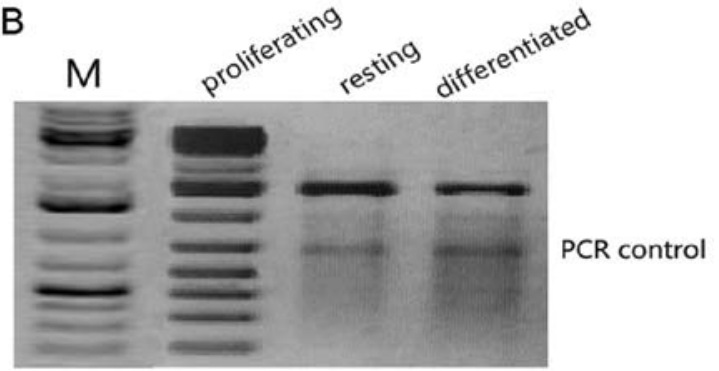

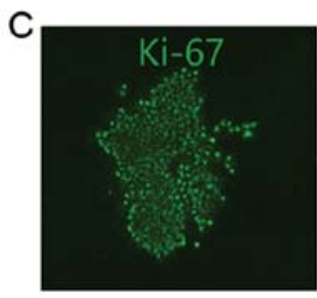
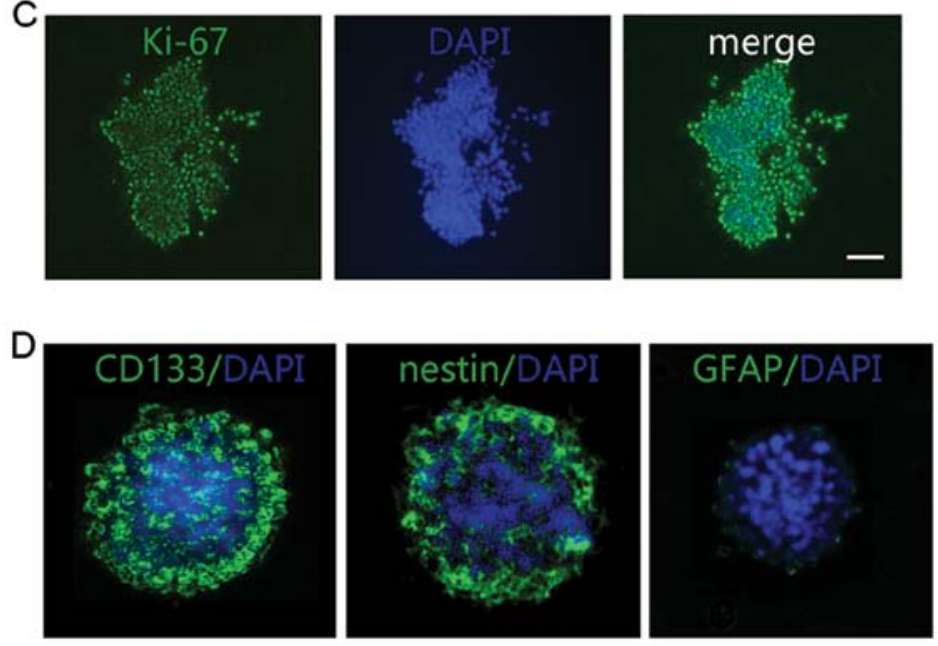

E
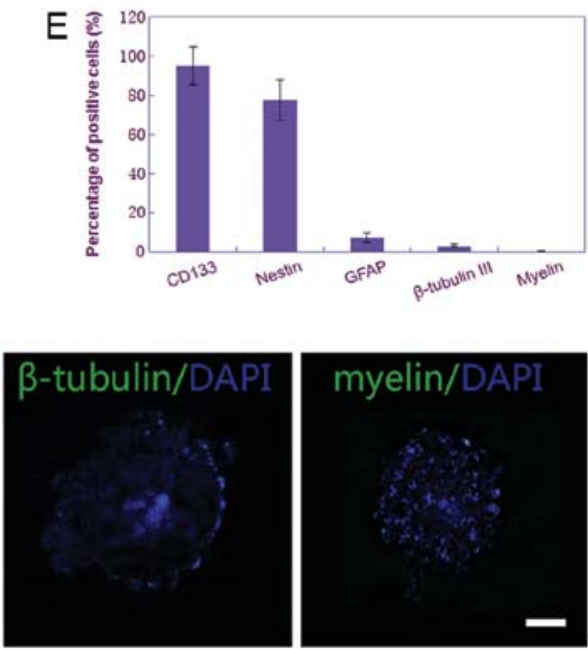

Figure 2. Proliferation and differentiation of CSGC spheres cultured on the surface of a soft gel in neurobasal medium with bFGF and EGF. (A) A schematic of culture of CSGC spheres. (B) Differentiation was detected using the telomeric repeat amplification assay. CSGC spheres expressed telomerase, as do other stem cell populations (lane 1). In contrast, resting or differentiated CGSCs were negative in terms of telomerase expression (lanes 2 and 3, respectively). (C) Immunocytochemical staining for Ki-67 showed that CSGCs cultured as described above were highly proliferative. (D) Immunocytochemical staining showed that most CSGC spheres were proliferating, thus not yet differentiated. (E) A bar chart showing the data of (D) (bars in micrographs, $25 \mu \mathrm{m})$.

but negative for the expression of CD31, CD34 and CD45 (Fig. 1F). While AT-MSCs were positive for the mesenchymal markers CD29, CD71, and CD105, but negative for the expression of CD34, CD45, and CD90 (Fig. 1G). Compared with BM- and AT-MSCs, the fibroblast cells displayed a relatively slow growth rate and a flat cell shape (Fig. 1E).

CSGCs in the proliferation status have strong tropism on MSCs. Because stiffness of cell adhesion substrates modulated stem cell proliferation and differentiation, we cultured the spheroids of CSGCs on the surface of the substrate with different stiffness, combined or not with bFGF and EGF in medium. The result of immunocytochemistry for Ki-67 showed that the CSGCs displayed highly proliferative activity when the CSGCs spheres were cultured on surface of the soft gel culture dishes with the Neurobasal medium containing bFGF and EGF (Fig. 2C). Further evidence for the CSGCs that were in the proliferation status was their expression of telomerase, as demonstrated in various other stem cell populations (28) (Fig. 2B). Moreover, immunocytochemical staining demonstrated that $\geq 90 \%$ of cells were positive for CD133 (marker for glioma stem cells) and $\sim 70 \%$ were positive for nestin (marker for glioma stem cells or neural precursor cells), whereas $<12 \%$ of cells were positive for GFAP (marker for glial differentiation), and $<5 \%$ were positive for $\beta$-tubulin III (marker for neuronal differentiation) and $<1 \%$ for myelin (marker for oligodendrocyte differentiation) (Fig. 2D and E). These results displayed that the CSGCs were in highly proliferative status when cultured on surface of the soft gel with the Neurobasal medium containing bFGF and EGF.

After the cells were incubated for $24 \mathrm{~h}$, the resulting conditioned media were collected and the chemoattractants were tested by ELISA. The results showed that the proliferating CSGCs could secreted much higher level of chemotactic factors into the culture supernatants, as tested SCF, SDF-1 and VEGF, compared with that in the resting or differentiated status, or the fibroblast cells (Fig. 3A-C). As chemoattractants, they could induce expression of respective receptor, c-Kit, CXCR4 and VEGFR, in both BM- and AT-MSCs (Fig. 3D-I). These findings were consistent with the results from the in vitro migration assay by transwell system, that displayed the maximum number of cell migration of BM- and AT-MSCs towards the supernatants (Fig. 4), compared with the homologous fibroblast cells. Taking into account the potential impact of bFGF and EGF, we set up control of the Neurobasal medium containing or lacking bFGF and EGF in the lower well of the Transwell plate. The results showed bFGF and EGF could slightly induce MSC migration, but insufficient to affect the experimental conclusion that the proliferating CSGCs have stronger tropism on BM- and AT-MSCs than in the resting and differentiated status (Fig. 4). 
A

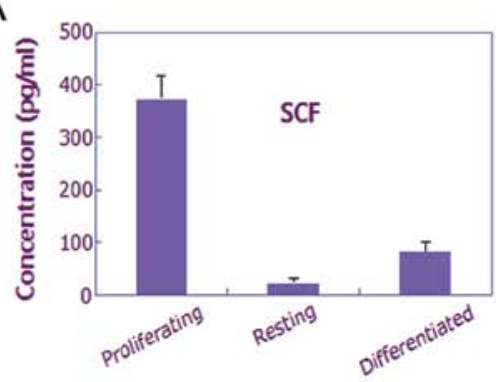

D

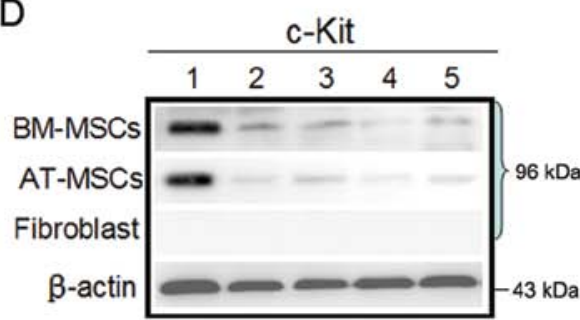

G

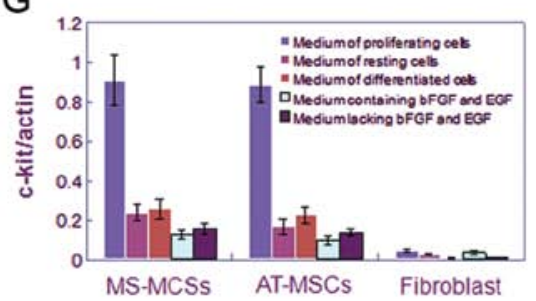

B

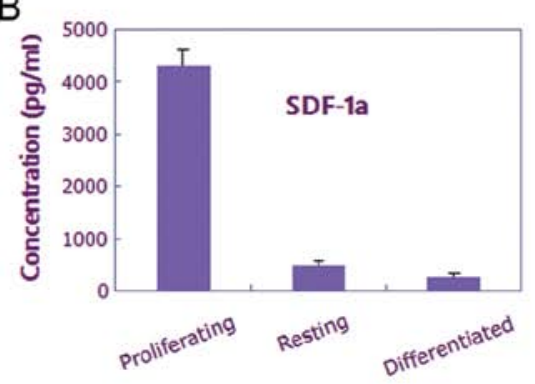

E

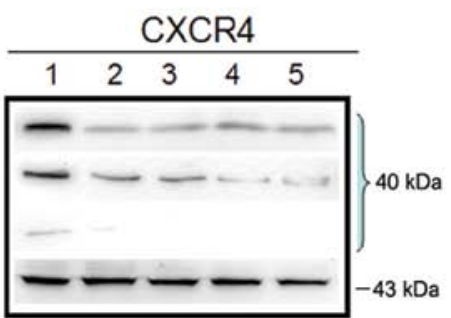

$\mathrm{H}$

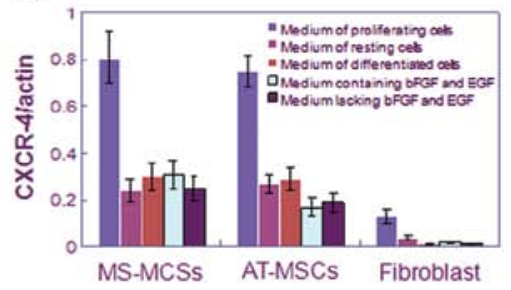

C

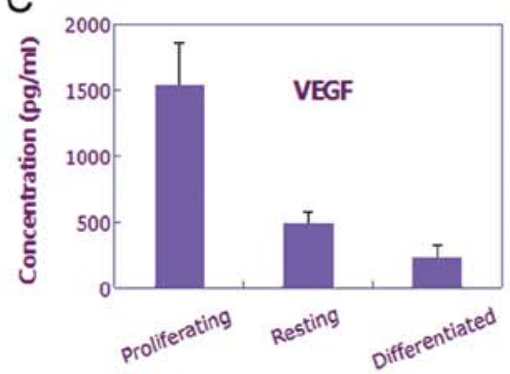

$\mathrm{F}$

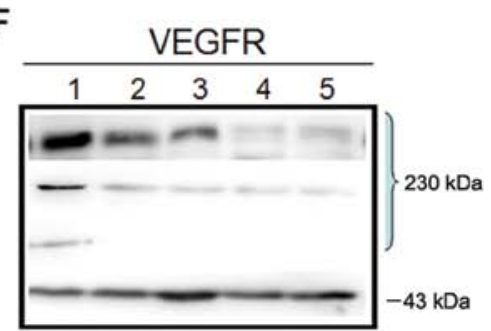

Figure 3. Assay of the expression levels of three cytokine/receptor pairs, SCF/c-Kit, SDF-1/CXCR4, and VEGF/VEGFR, in media conditioned by CSGCs, MSCs, and fibroblast cells. (A-C) The levels of three cytokines, SCF, SDF-1, and VEGF, were measured by ELISA in CSGC medium when CSGCs were cultured under different conditions. Only proliferating CSGCs expressed SCF, SDF-1 and VEGF at high levels. (D-F) Western blotting was used to measure the levels of three cytokine receptors, c-Kit, CXCR4, and VEGFR, in MSCs and fibroblasts. (G-I) Bar charts showing the data of (D-F). BM- and AT-MSCs expressed c-Kit, CXCR4, and VEGFR at high levels only when cultured in medium conditioned by proliferating CSGCs, thus not in medium conditioned by resting or differentiated CSGCs. Fibroblasts displayed only slight tropism toward proliferating CSGCs.

A

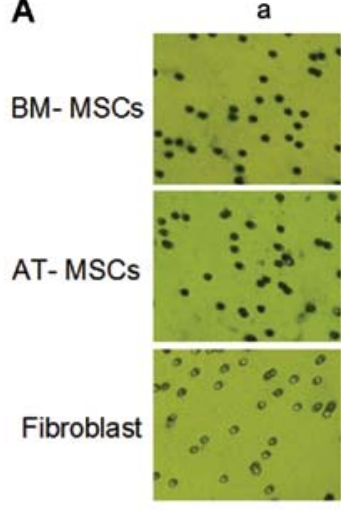

B

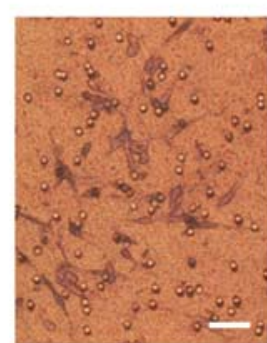

b

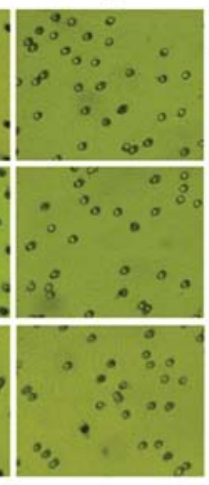

c

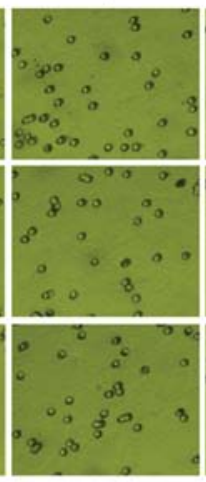

C

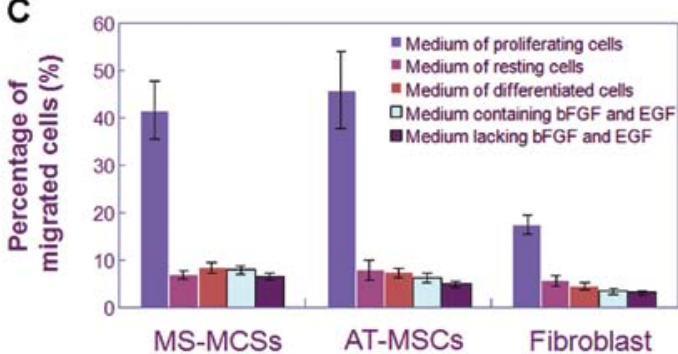

Figure 4. In vitro migration studies. The migratory capacities of BM- and AT-MSCs were determined using Transwell plates. (A) BM- and AT-MSCs migrated more strongly toward medium conditioned by proliferating CSGCs than toward medium conditioned by resting or differentiated CSGCs. (B) Some cells migrated through the pore filters of the Transwell plates. (C) A bar chart showing the data of a (bar in micrograph, $15 \mu \mathrm{m}$ ). 
A

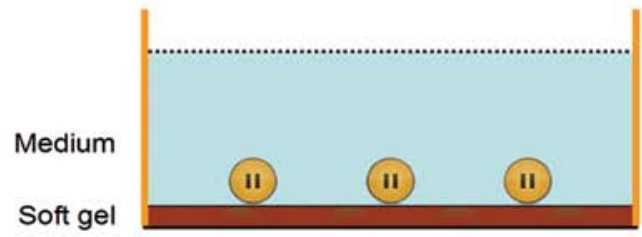

E

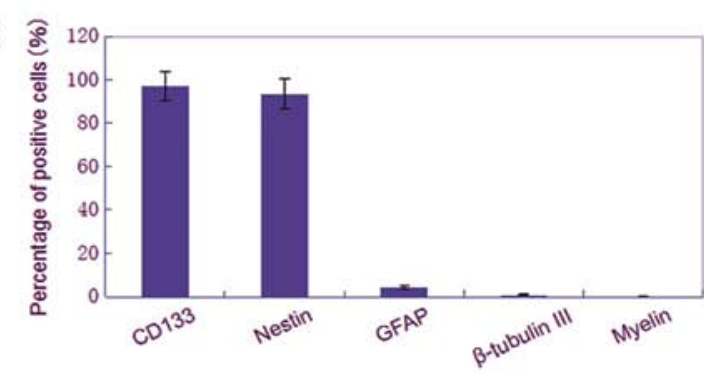

B

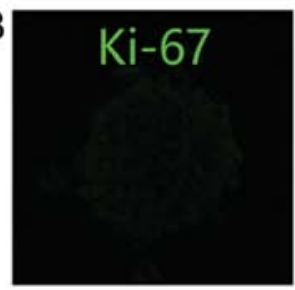

C $\mathrm{Ki}-67$

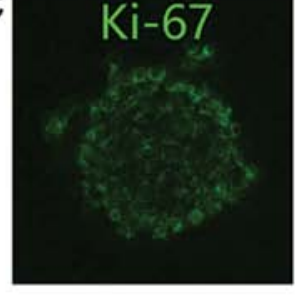

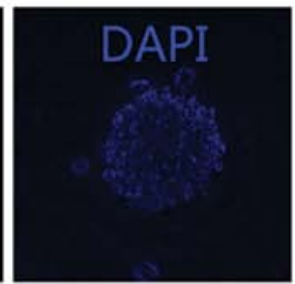
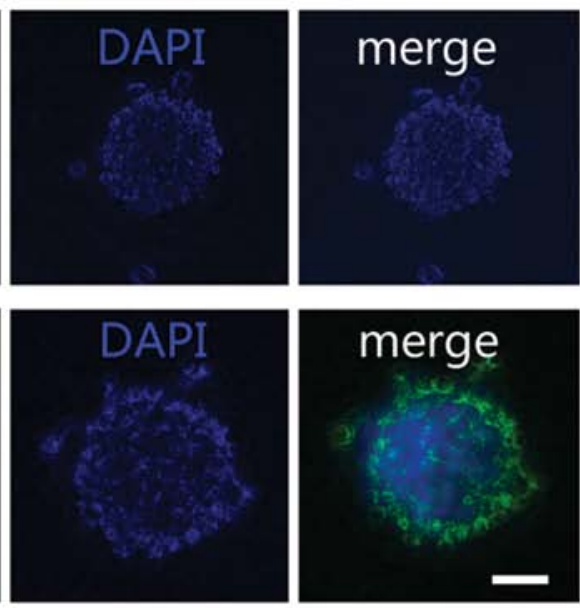
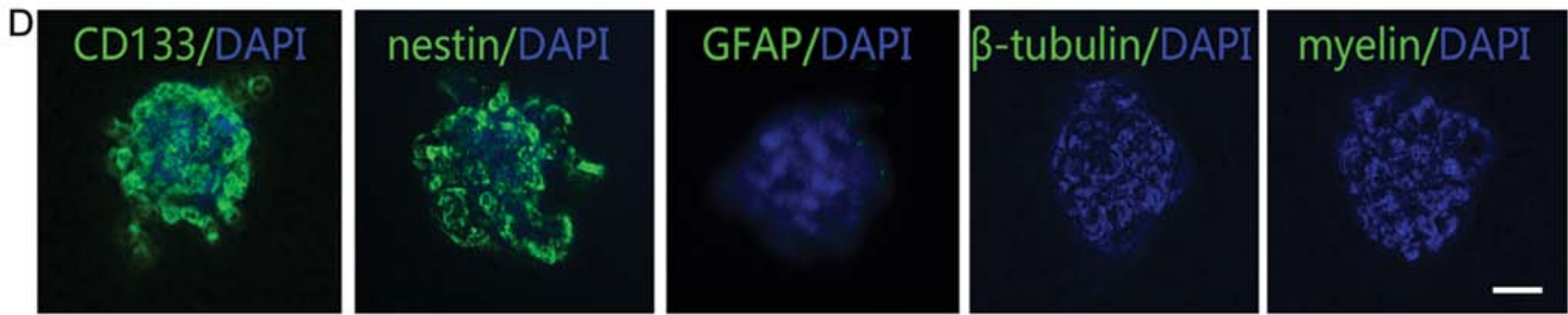

Figure 5. The proliferation and differentiation of CSGC spheres cultured on a soft gel in neurobasal medium lacking bFGF and EGF. (A) A schematic of the culture conditions used. (B) Staining for Ki-67 revealed that CSGCs displayed low proliferative activity when cultured as described above. (C) When bFGF and EGF were added to the medium, CSGCs resumed rapid proliferation, as evidenced by expansion of the CSGC spheres and increased Ki-67 positivity. (D) Immunocytochemical staining showed that most CSGCs were undifferentiated. (E) A bar chart showing the data of (D) (bars in micrographs, $25 \mu \mathrm{m}$ ).

A

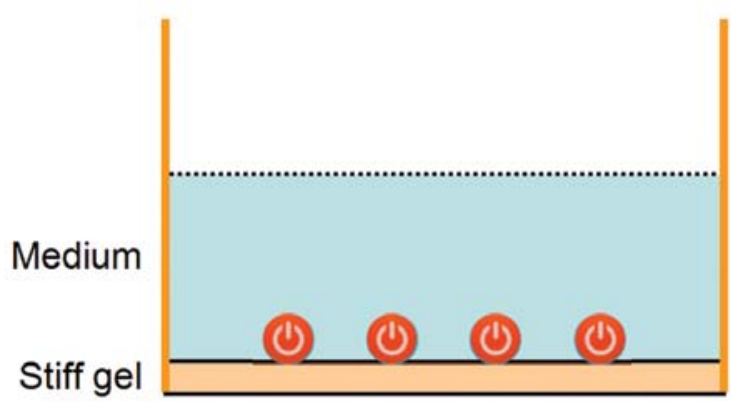

C

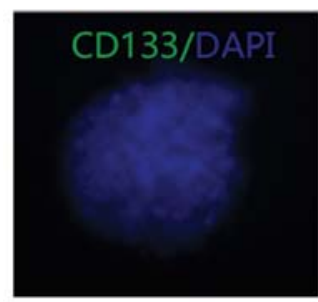

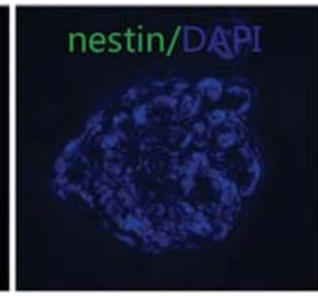

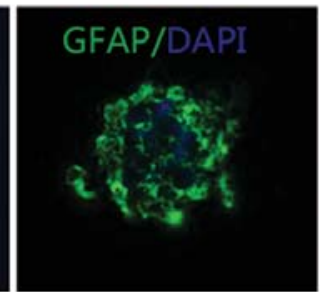

B
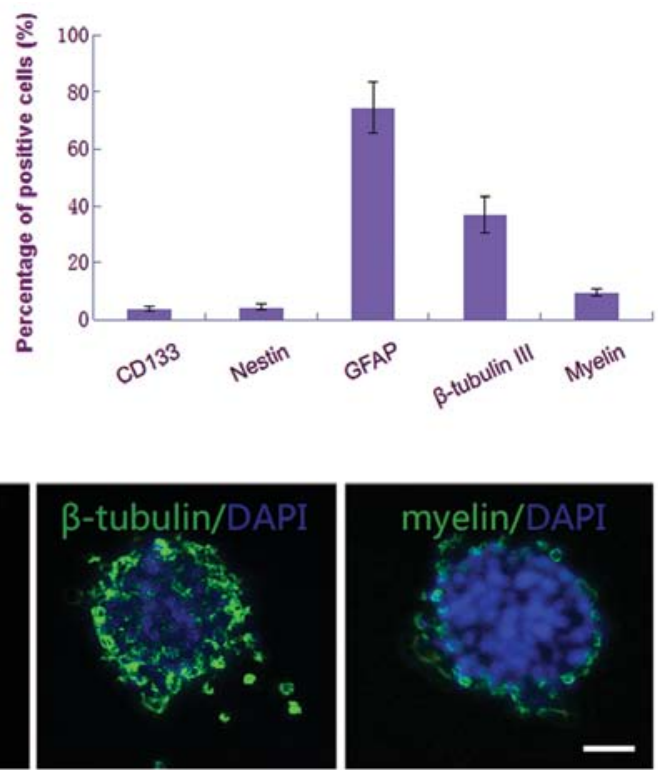

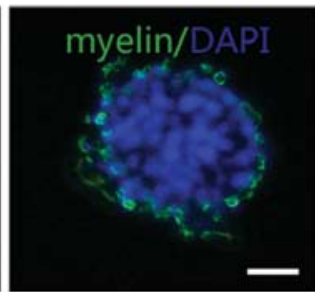

Figure 6. Proliferation and differentiation of CSGC spheres cultured on the surface of a stiff gel in neurobasal medium lacking bFGF and EGF. (A) A schematic drawing showing the culture conditions used. (B) Immunocytochemical staining showed that most CSGC cells were differentiated. (C) A bar chart showing the data of (D) (bars in micrographs, $25 \mu \mathrm{m}$ ).

CSGCs in the resting status have little tropism on MSCs. When cultured on surface of the soft gel culture dishes with the same Neurobasal medium but lacking bFGF and EGF, the CSGCs spheres showed growth arrest. Almost no cells were positive for Ki-67 staining (Fig. 5B). The results of immunocytochemical staining demonstrated that $\sim 97 \%$ of cells were positive for $\mathrm{CD} 133$ and $\sim 90 \%$ were positive for nestin, whereas $<6 \%$ of cells were positive for GFAP, and $<2 \%$ were positive 
for $\beta$-tubulin III and myelin (Fig. 5D and E). These results confirmed most of the CSGCs were in resting status. When re-adding bFGF and EGF in medium, these CSGCs restored the rapid proliferation rate, with the performance of expanding CSGC spheres and increasing positive staining for Ki-67 (Fig. 5C).

The results of migration assay showed there was scarcely any cell migration of BM- and AT-MSCs towards the culture supernatants (Fig. 4), compared with the homologous fibroblast cells. These results were consistent with the findings of ELISA assay, that showed the expression of SCF, SDF-1 and VEGF at very low level (Fig. 3A-C), and the results of western blotting that displayed little protein express of c-Kit, CXCR4 and VEGFR in both BM-MSCs and AT-MSCs (Fig. 3D-I).

Differentiated CSGCs have little tropism on MSCs. To induce differentiation of CSGCs into terminal cells, the CSGC spheres were cultured on surface of the stiff gel culture dishes with the same Neurobasal medium but lacking bFGF and EGF. The result of telomeric repeat amplification protocol assay showed obvious regression of telomerase activity (Fig. 2B), as well as the cell differentiation of CSGCs into terminal cells tested by immunocytochemistry. In contrast to the CSGCs that were in the proliferation status, the differentiated cells of these spheroids were negative for CD133 and nestin, with high level expression of GFAP, $\beta$-tubulin III, and myelin (Fig. 6B and C).

Like the cells in the resting status, the differentiated CSGCs have little tropism on either BM-MSCs or AT-MSCs, with fewer migrating cells (Fig. 4), lower level expression of the cytokine/receptor pairs, SCF/c-Kit, SDF-1/CXCR4 and VEGF/VEGFR, compared with the CSGCs in the proliferation status (Fig. 3).

CSGCs in the proliferation status show slight tropism on fibroblast cells. In this study, fibroblast, as a negative control, also showed slight tropism towards the proliferating CSGCs, but not the resting and the differentiated CSGCs (Fig. 4), consistent with the characteristic of weak expression of the receptors of CXCR4 and VEGFR (Fig. 3D-I). This finding seems to be contradictory to previous reports, which claimed fibroblasts have hardly any tropism for tumor cells. We speculate that fibroblasts may be involved in the cancer stem cell metastasis as a helper cell component.

\section{Discussion}

MSCs can differentiate into a variety of adult mesenchymal tissues including bone, cartilage, adipose, muscle, ligament, and tendon, and seem to be a promising source for tissue repair and gene therapy (29). In the past decade, emerging studies show MSCs can be envisioned as vehicles for both short-term and long-term gene therapy, as MSCs are easily transduced by a variety of vectors with various therapeutic drugs (30). In brain tumor gene therapy, MSCs demonstrate acceptable therapeutic effect. This is attributable to the fundamental ability of MSCs to migrate, or home, to brain tumors irrespective of the blood-brain barrier (BBB) and to be manipulated into expressing various therapeutic molecules (31). However, many challenges exist. The key issues include: i) Brain tumors exhibit phenotypic heterogeneity, being composed of cells: a) resting stem cells and b) proliferating cells with limited self-renewal, and c) terminal cells: either resting differentiated cells or apoptotic cells. It is unclear whether all the tumor cells may induce MSCs migrating. ii) Most chemotherapeutic drugs are toxic preferentially to proliferating cells. However, most tumor stem cells are resting, that offers the possibility that cancer cells escape chemotherapyand radiotherapy-induced cell death, and contribute to local tumor recurrence (32).

In this study we tested the tropism character of two MSC cell lines, the BM- and AT-MSCs, toward the resting, proliferating or differentiated CSGCs for the first time. In order to define the three states of cells, we cultured the spheroids of CSGCs on the surface of the substrate with different stiffness, combined with or without bFGF and EGF in the medium. Study of Park et al confirmed that stiffness of cell adhesion substrates modulated stem cells proliferation and differentiation. Stem cells on soft substrates had less spreading, fewer stress fibers and lower proliferation rate than those on stiff substrates (33). Others reported glioblastoma multiformderived cell lines expanded slowly in the presence of the only FGF2, switched to a faster growth mode when exposed to EGF alone, and expanded even faster when exposed to both mitogens simultaneously (34). Thus, we tested the proliferation and differentiation status of CSGCs by combining application of the substrate with different stiffness with the culture medium containing or not bFGF and EGF. The experimental results bear out our supposition. When cultured on surface of the soft gel culture dishes with the Neurobasal medium containing bFGF and EGF, the spheroids of CSGCs displayed highly proliferative activity, with strong Ki-67 staining, high expression of telomerase, and extremely low differentiation into terminal nerve cell lines, such as neurons, astrocytes, and oligodendrocytes. Whereas, when cultured on surface of the soft gel culture dishes with the medium lacking bFGF and EGF, the spheroids of CSGCs were still in resting status, with changes continuing till the presence of FGF2 and EGF again. Lastly, when cultured on surface of the stiff gel culture dishes with the medium lacking bFGF and EGF, the spheroids of CSGCs differentiated into terminal cell lines.

Furthermore, we tested the tropism of MSCs toward the resting, proliferating or differentiated CSGCs by transwell migration system. Taking into account that heterologous cells may affect results of cell tropism, we obtained BM- and AT-MSCs, fibroblast cells, and CSGCs from homologous tumor-bearing mice, because homologous cells show the lowest immunogenicity. The results of in vitro migration test showed that the proliferating CSGCs displayed the strongest tropism on both BM- and AT-MSCs, whereas, there were few MSC migrating towards CSGCs that were in the resting and differentiated status.

It is known that the migration of MSCs in vitro was stimulated by conditioned medium from cultured glioma cells in a dose-dependent manner. This indicates that soluble factors released from glioma cells could induce the migration of MSCs. However, these brain tumor stem cells inducing migratory properties require further elucidation. In this study, we detected, for the first time, the level of three key 
cytokines, namely SCF, SDF-1, and VEGF, secreted by the proliferating, resting, and differentiated CSGCs. Concurrent with the results of in vitro migration, the highest level expression of the three cytokines was found in the proliferating CSGCs compared to that in resting, and differentiated CSGCs. These cytokines further induced expression of their respective receptors, c-Kit, CXCR4 and VEGF, on both BM-MSCs and AT-MSCs. These findings showed that BMand AT-MSCs have little migration towards CSGCs that were in the resting and differentiated status. Only when induced into proliferation process, CSGCs secrete chemokines and induce migration of MSCs.

Moreover, we found, in the present study, that the proliferating CSGCs showed slight tropism also on fibroblasts. This finding seems to be contradictory with previous reports, which claimed fibroblasts have hardly any tropism for tumor cells. We speculate that fibroblasts may be involved in the cancer stem cells metastasis as a helper cell component.

The essential significance of this study lies in that it gives new insights into glioma treatment using MSCs as vectors with various therapeutic drugs. The cancer stem cells, a minority population within the tumor, play a key role in tumor recurrence. A satisfactory clinical application is only achieved when eliminating all cancer cells, especially the cancer stem cells, by efficient targeting gene therapy strategy. In our study, we found that CSGCs, when in resting status, have little tropism on MSCs, limiting the application of MSCs as drug vectors. Furthermore, most therapeutic drugs play a role in targeting the tumor cells in the cell cycle. Thus, it is important to find an alternative way to induce the resting cancer stem cells into proliferating status, before or during using the tracking ability of MSCs to kill all tumor cells.

\section{Acknowledgements}

This study was supported by the China National Natural Scientific Fund (81000901), Key Laboratory Project of Tianjin Programs for Science and Technology (10SYSYJC28800), Key Project of Chinese National Programs for Fundamental Research and Development (973 Program, 2010CB529405), and Tianjin Health Bureau Science and technology projects (2011KZ24 and 2011KZ26), Tianjin Binhai New Area Health Bureau Science and technology projects (2011BHKZ004).

\section{References}

1. Raizer JJ, Grimm S, Chamberlain MC, et al: A phase 2 trial of single-agent bevacizumab given in an every-3-week schedule for patients with recurrent high-grade gliomas. Cancer 116: $5297-5305,2010$

2. Gilbert CA and Ross AH: Cancer stem cells: cell culture, markers, and targets for new therapies. J Cell Biochem 108: 1031-1038, 2009

3. Bonavia R, Inda MM, Cavenee WK and Furnari FB: Heterogeneity maintenance in glioblastoma: a social network. Cancer Res 71: 4055-4060, 2011.

4. Masica DL and Karchin R: Correlation of somatic mutation and expression identifies genes important in human glioblastoma progression and survival. Cancer Res 71: 45504061, 2011.

5. Huang PH, Cavenee WK, Furnari FB and White FM: Uncovering therapeutic targets for glioblastoma: a systems biology approach. Cell Cycle 6: 2750-2754, 2007.
6. Hide T, Takezaki T, Nakatani Y, et al: Combination of a ptgs2 inhibitor and an epidermal growth factor receptor-signaling inhibitor prevents tumorigenesis of oligodendrocyte lineagederived glioma-initiating cells. Stem Cells 29: 590-599, 2011.

7. Murphy AM and Rabkin SD: Current status of gene therapy for brain tumors. Transl Res 161: 339-354, 2013.

8. Gong $\mathrm{A}$ and Huang S: FoxM1 and Wnt/ $\beta$-catenin signaling in glioma stem cells. Cancer Res 72: 5658-5662, 2012.

9. Filatova A, Acker T and Garvalov BK: The cancer stem cell niche(s): the crosstalk between glioma stem cells and their microenvironment. Biochim Biophys Acta 1830: 2496-2508, 2013.

10. Altaner C and Altanerova V: Stem cell based glioblastoma gene therapy. Neoplasma 59: 756-760, 2012.

11. Patel M, Vogelbaum MA, Barnett GH, Jalali R and Ahluwalia MS: Molecular targeted therapy in recurrent glioblastoma: current challenges and future directions. Expert Opin Investig Drugs 21: 1247-1266, 2012

12. Serakinci N, Christensen R, Fahrioglu U, Sorensen FB, DagnæsHansen F, Hajek M, Jensen TH, Kolvraa S and Keith NW: Mesenchymal stem cells as therapeutic delivery vehicles targeting tumor stroma. Cancer Biother Radiopharm 26: 767-773, 2011.

13. Bak XY, Lam DH, Yang J, Ye K, Wei EL, Lim SK and Wang S: Human embryonic stem cell-derived mesenchymal stem cells as cellular delivery vehicles for prodrug gene therapy of glioblastoma. Hum Gene Ther 22: 1365-1377, 2011.

14. Dai LJ, Moniri MR, Zeng ZR, Zhou JX, Rayat J and Warnock GL: Potential implications of mesenchymal stem cells in cancer therapy. Cancer Lett 305: 8-20, 2011.

15. Hata N, Shinojima N, Gumin J, Yong R, Marini F, Andreeff M and Lang FF: Platelet-derived growth factor BB mediates the tropism of human mesenchymal stem cells for malignant gliomas. Neurosurgery 66: 144-157, 2010.

16. Matuskova M, Baranovicova L, Kozovska Z, Durinikova E, Pastorakova A, Hunakova L, Waczulikova I, Nencka R and Kucerova L: Intrinsic properties of tumour cells have a key impact on the bystander effect mediated by genetically engineered mesenchymal stromal cells. J Gene Med 14: 776-787, 2012.

17. Keung EZ, Nelson PJ and Conrad C: Concise review: genetically engineered stem cell therapy targeting angiogenesis and tumor stroma in gastrointestinal malignancy. Stem Cells 31: 227-235, 2013.

18. Kosaka H, Ichikawa T, Kurozumi K, Kambara H, Inoue S, Maruo T, Nakamura K, Hamada H and Date I: Therapeutic effect of suicide gene-transferred mesenchymal stem cells in a rat model of glioma. Cancer Gene Ther 19: 572-578, 2012.

19. Doucette T, Rao G, Yang Y, Gumin J, Shinojima N, Bekele BN, Qiao W, Zhang W and Lang FF: Mesenchymal stem cells display tumor-specific tropism in an RCAS/Ntv-a glioma model. Neoplasia 13: 716-725, 2011.

20. Liu Y, Wang L, Fatahi R, Kronenberg M, Kalajzic I, Rowe D, $\mathrm{Li} \mathrm{Y}$ and Maye P: Isolation of murine bone marrow derived mesenchymal stem cells using Twist 2 Cre transgenic mice. Bone 47: 916-925, 2010.

21. Kisiel AH, McDuffee LA, Masaoud E, Bailey TR, Esparza Gonzalez BP and Nino-Fong R: Isolation, characterization, and in vitro proliferation of canine mesenchymal stem cells derived from bone marrow, adipose tissue, muscle, and periosteum. Am J Vet Res 73: 1305-1317, 2012.

22. Dembo M and Wang YL: Stresses at the cell-to-substrate interface during locomotion of fibroblasts. Biophys $\mathrm{J} 76$ : 2307-2316, 1999.

23. Qin L, Huang J, Xiong C, Zhang Y and Fang J: Dynamical stress characterization and energy evaluation of single cardiac myocyte actuating on flexible substrate. Biochem Biophys Res Commun 360: 352-356, 2007.

24. Huang J, Peng X, Xiong C and Fang J: Influence of substrate rigidity on primary nucleation of cell adhesion: a thermal fluctuation model. J Colloid Interface Sci 366: 200-208, 2012.

25. Lee J, Elkahloun AG, Messina SA, Ferrari N, Xi D, Smith CL, Cooper R Jr, Albert PS and Fine HA: Cellular and genetic characterization of human adult bone marrow-derived neural stem-like cells: a potential antiglioma cellular vector. Cancer Res 63: 8877-8889, 2003.

26. Paroo Z, Ye X, Chen S and Liu Q: Phosphorylation of the human microRNA-generating complex mediates MAPK/Erk signaling. Cell 139: 112-122, 2009. 
27. Liu N, Landreh M, Cao K, et al: The microRNA miR-34 modulates ageing and neurodegeneration in Drosophila. Nature 482: 519-523, 2012.

28. Bischoff DS, Makhijani NS and Yamaguchi DT: Constitutive expression of human telomerase enhances the proliferation potential of human mesenchymal stem cells. Biores Open Access 1: 273-279, 2012.

29. Sensebé L, Bourin P and Tarte K: Good manufacturing practices production of mesenchymal stem/stromal cells. Hum Gene Ther 22: 19-26, 2011.

30. Boeuf S and Richter W: Chondrogenesis of mesenchymal stem cells: role of tissue source and inducing factors. Stem Cell Res Ther 1: 31, 2010.

31. Uccelli A, Morando S, Bonanno S, Bonanni I, Leonardi A and Mancardi G: Mesenchymal stem cells for multiple sclerosis: does neural differentiation really matter? Curr Stem Cell Res Ther 6: 69-72, 2011.
32. Augello A and De Bari C: The regulation of differentiation in mesenchymal stem cells. Hum Gene Ther 21: 1226-1238, 2010.

33. Park JS, Chu JS, Tsou AD, Diop R, Tang Z, Wang A and Li S: The effect of matrix stiffness on the differentiation of mesenchymal stem cells in response to TGF- $\beta$. Biomaterials 32: 3921-3930, 2011.

34. Galli R, Binda E, Orfanelli U, Cipelletti B, Gritti A, De Vitis S, Fiocco R, Foroni C, Dimeco F and Vescovi A: Isolation and characterization of tumorigenic, stem-like neural precursors from human glioblastoma. Cancer Res 64: 7011-7021, 2004. 